\title{
Delivering 3D Advertising to Mobile Phones
}

\author{
Fadi Chehimi, Paul Coulton and Reuben Edwards
}

f.chehimi, p.coulton, and r.edwards@lancaster.ac.uk

\begin{abstract}
Directing advertising to mobile phones currently is limited to commercial text messages, short-code text-back messages, two dimensional (2D) images, or Wireless Access Protocol (WAP) clickable push links. All of these traditional methods do not facilitate advertising approach were consumers can interact with prospective purchases. In this paper we introduce a novel and highly interactive location- and permissionbased advertising system that allows 3D product adverts to be displayed on users' mobile phones. The paper provides a thorough discussion of the system covering its performance, implementation structure, platform-dependent optimizations and suggestions for future work. With mobile phones and 3D interactive tools, advertising becomes more engaging, rewarding and entertaining and provides marketing executives with new means of directing their campaigns to a more specific target audience.
\end{abstract}

\section{INTRODUCTION}

The general public is increasingly using the Internet for electronic commerce (e-commerce) activities. The emergence of mobile phones as ubiquitous consumer devices will extend their range of activities into mobile commerce. M-commerce can be defined as a set of business activities conducted over cellular or wireless networks [1]. It not only includes ecommerce applications on mobile platforms, but also many new and innovative applications such as mobile advertising.

Mobile advertising is not simply linking users to websites by clicking on banners or pop-up windows through their mobile phone browsers. It offers the ability to go beyond the mainstream of electronic advertising, which mainly targets the mass market predominantly over the Internet, to allow businesses to deliver their particular messages to specific individuals at specific locations. Mobile phones will enable a greater range of application delivery, deeper consumer interaction (and hence better data collection), and greater and faster consumer reach [2]. Further, as mobile phones are heavily used by the 15-24 demographic, which is generally considered the hardest to reach [3], advertising on this medium will present marketers with a new, attractive and familiar technology for reaching not only this particular group but any user of a mobile phone.

Mobile adverts can come in a variety of forms although primarily they are simple text-based messages or simple twodimensional (2D) graphics. With the increasing availability of standardized operating systems [4] and improved graphical capabilities [5] there are exciting possibilities for the creation of three-dimensional (3D) mobile advertisements. In this paper we shall present a novel system capable of delivering and viewing such advertisements. We will address the issue of privacy which is obviously a concern for the public [6] by ensuring that potential customers are required to opt-in rather than opt-out of the service.

The system presented in this paper allows both a location and permission-based advertising designed to provide an onthe-spot 3D interactive mobile adverts on mobile phones which we have termed $3 D M-A d$. Users choose which product to receive, and then view, from a list of products presented on large public display screens which can be either co-located within a particular business or be a virtual business in the form of a stand alone display (possibly located at entrances of shopping malls, airport duty-frees, restaurants or cafes). Both possibilities require a back-end infrastructure that controls the interaction with the potential customers and the delivery of the 3D adverts. Should they wish to accept these adverts or request details of a particular product the system will transmit this information to customers' mobile phones via Bluetooth. The software on the phone will then list all items they have previously received as shown in Fig. 1a. In this way customers can keep 3D copies of products they like on their phones for later retrieval or alternatively transmit them to friends or family to comment and view prospective purchases remotely. The 3D adverts in the screenshots shown in Figs. 1b, 1c, 1d and 1e display a sample product (Ancient Roman Vase) interactively from all angles, thus providing a greater amount of details than can be presented in $2 \mathrm{D}$ images. As shown in the figures, the user can rotate, move horizontally/vertically or zoom in/out the product for better viewing experience.

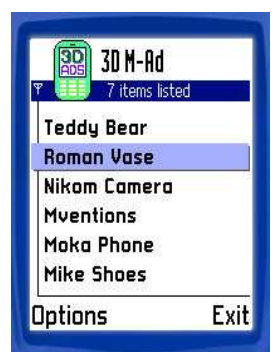

1a: Products List

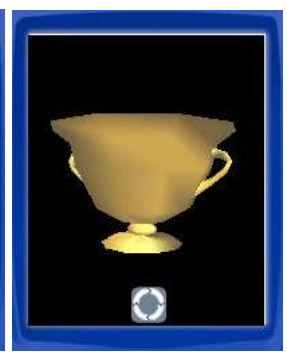

1b: Initial view

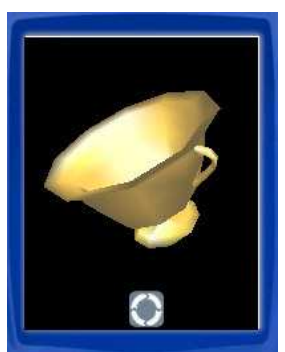

1c: Rotated view

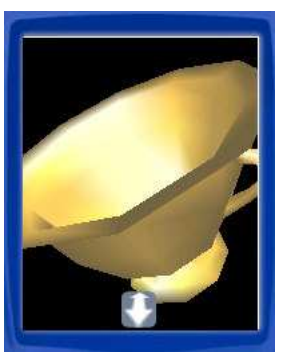

1d: Zoomed in view

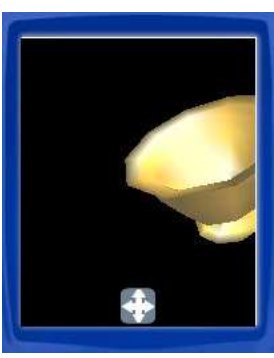

1e: Side movement view
Fig. 1: Listing and viewing products on phones 
This advertising system allows customers to retrieve additional information about a product without having to relocate to the particular business advertising it; or to obtain information when the business might be closed as shown in Fig. 2. Once the purchase decision has been taken by the consumer, and -most likely- his friends and family, he can make the purchase remotely through General Packet Radio Service (GPRS) connection to the business's server through a secure payment mechanism. When the payment is received, the product will be automatically dispatched to the address registered with the payment details, unless the user specifies an alternative.



Fig. 2: Roman Vase Product Information

This unique marketing behavior will help create the socalled "buzz" that marketers often seek for a product which will empower their ability to produce viral-marketing campaigns.

\section{SYSTEM INFRASTRUCTURE}

3D M-Ad system consists of a client/server architecture with the client residing on the mobile phone and the server representing the business (real or virtual). The client software, $3 D$ M-Ad Viewer, is currently developed for mobile phones running Symbian mobile operating system, and more particularly Series 60. As 25 million Series 60 devices have been shipped by Nokia by the end of May 2005 [7] and more than 250 million smartphones (a combination of cellular phone and handheld computer [8]) expected to be shipped by 2008 [9], there will be a potential market base for this system. In order to widen the proliferation of this system corresponding software can be developed for suitable Brew or Java enabled mobile phones.

3D M-Ad Viewer is used to request and display products along with their commercial/promotional details and enables the sharing of information via either Bluetooth or Multimedia Message Service (MMS) with other users. The software facilitates payments from the mobile phone to the business via a secure GPRS connection.

3D M-Ad Manager, which is the server side of the systems, provides management facilities to the business so that new products may be added or existing products may have their information updated. Every product is represented by a textual data file containing its 3D geometrical representation and commercial information, which is generated by the management system, and a texture image giving the product's outer appearance.

\section{SYSTEM FUNCTIONALITY}

Products' data files are transmitted to any Bluetooth-enabled mobile phone in the vicinity of the server by 3D M-Ad Manager once requested by the customer via the 3D M-Ad Viewer installed on his mobile phone. The user is then prompted to either accept or reject the adverts by the built-in Bluetooth protocol. This confirmation forms a second layer of verification to enable opting-in to the service. The data files, if accepted, can only be viewed by the 3D M-Ad Viewer which is transmitted to mobile phones via the same server infrastructure if it has not been previously installed. Fig. 3 defines the system operations, at client and server, when transmitting product data-files and/or the 3D M-Ad Viewer.

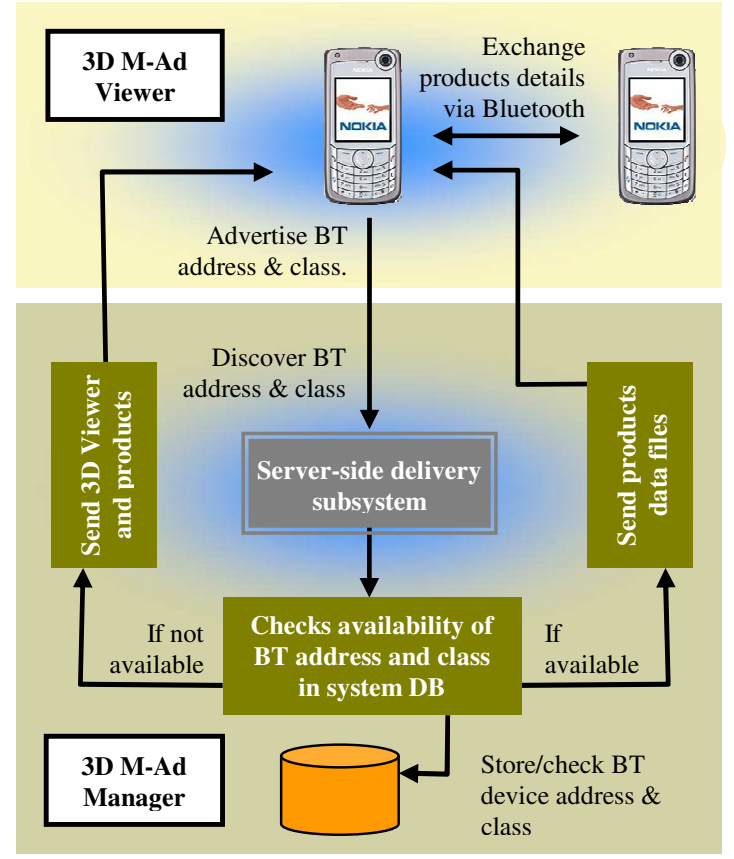

Fig. 3: Client/Server Architecture for 3D M-Ad

Information of previous product installations are stored in the server database which is referenced by Bluetooth addresses of devices that accepted software installations in the past. The server is constantly trying to discover nearby Bluetoothenabled devices and compares them to entries within its database for identification. If a device's Bluetooth address is not available, the server will initially try to send the 3D M-Ad Viewer. This transmission process to non-registered potential customers is performed only once per hour to that particular address to ensure that they are not harassed with infinite number of notifying messages every time they pass near within range of the server. After having the software installed, the customer may download the desired product by typing its ondisplay code and sending it to the server.

Discovering Bluetooth devices' classes is required here to enable the system to limit the transmission process to mobile phones and avoid connecting to other Bluetooth devices like desktops/laptops, audio headsets, LAN ho 